\title{
Recurrent Primary Vitreoretinal Lymphoma
}

National Cancer Institute

\section{Source}

National Cancer Institute. Recurrent Primary Vitreoretinal Lymphoma. NCI Thesaurus.

Code C157068.

The reemergence of primary vitreoretinal lymphoma after a period of remission. 